\title{
Responsive Surface Design to Reduce the Urban Heat Island Effect (UHI)
}

\author{
Gibsy Marcela Estrada Calderón \\ Universidad Michoacana de San Nicolás de Hidalgo | México | gibsyes@gmail.com \\ Habid Becerra Santacruz \\ Universidad Michoacana de San Nicolás de Hidalgo | México | hbecerra@umich.mx
}

\begin{abstract}
The present project aims to influence the reduction of the urban heat island effect (UHI) by designing sensitive surfaces that respond to changes in temperature. This research presents a scenario with a vision of cities with adaptive designs that is generated from the insertion of elements sensitive to the environment (elements that respond to environmental stimuli). Responsive elements become constant factors in small-scale or large-scale design that transform the way environment is changed to a sensitive and resilient urban environment against possible adverse environmental conditions.
\end{abstract}

Keywords: Urban heat island; Surface design; Sensitive environments; Resilient.

\section{INTRODUCCIÓN}

A través de la historia, el ser humano ha tenido la necesidad de asentarse en lugares que cuentan con recursos suficientes para desarrollarse, relacionarse y conformar una sociedad. Estos asentamientos humanos al pasar del tiempo inciden en el cambio de las condiciones del entorno natural. Áreas naturales se convierten en zonas rurales, y estas a su vez en ciudades. A partir de los años 70's el crecimiento de las ciudades ha sido exponencial, el cambio climático se ha convertido en un tema global, a partir de esto se comienza a considerar el espacio urbano como algo que se tendría que planear y regular.

Sin embargo, las condiciones económicas y sociales, así como la velocidad y forma, en las que crece la mancha urbana, dificultan la posibilidad de aplicar planes estratégicos de crecimiento urbano, por lo que en la mayoría de los casos es necesaria la aplicación de estrategias correctivas en el diseño de las ciudades. Se espera que para el año 2050 el $70 \%$ de la población viva en zonas urbanas, (United Nations, 2018) lo que significa que las superficies modificadas también aumentarán.

La ciudad actúa como un elemento que modifica el clima de la región, este clima generado por la ciudad lo podemos definir como microclima, el cual responde a condiciones específicas que están directamente relacionadas con la morfología, estructura y condiciones de una ciudad.

El efecto de isla de calor urbana se genera por varios factores, el aumento de áreas urbanizadas, una planeación urbana carente de la aplicación de estrategias de sostenibilidad para la ciudad, la utilización de materiales poco sustentables y $/ 0$ adecuados para superficies de vialidades, estacionamientos, etcétera. Las islas de calor urbano pueden provocar problemas de confort tanto en espacios exteriores como interiores y esto genera que haya mayor uso energético para enfriar espacios en verano o calentarlo en invierno.

Este problema también representa un riesgo para la salud, sobre todo para los sectores más vulnerables como niños y ancianos, así como problemas económicos y el ya mencionado energético. (Tumini, 2010)

La isla de calor urbana es la respuesta de muchos factores, los cuales pueden ser catalogados como controlables e incontrolables. A su vez estos factores pueden ser clasificados como variables temporales en el tiempo, por ejemplo, la velocidad del viento o la cobertura de las nubes; como variables constantes en el tiempo como lo son las zonas verdes, el material de los edificios y el factor de visión del cielo; y como variables cíclicas como la radiación solar o las fuentes de calor antropogénico. (Rizwan et al., 2008)

Se pueden distinguir dos tipos de ICU (Isla de Calor Urbana), la superficial y la atmosférica, que según la investigadora Irina Tumini, se diferencian por los elementos que la generan, los métodos para identificarlas y medirlas, los impactos generados y en algunos casos, las formas para mitigarlas. La isla de calor superficial se genera por la elevación de la temperatura de superficies de suelos, techos y fachadas, esta temperatura se registra mayor a la temperatura del aire.

La isla de calor superficial se puede producir de igual forma en el día como en la noche, pero tiende a ser más fuerte durante el día y con el cielo despejado. La isla de calor atmosférica se determina por la diferencia de temperatura 
del aire entre las zonas urbanas y las zonas rurales. (Rizwan et al., 2008)

Existe una relación entre el microclima y el uso que se le da a los espacios públicos, esto de acuerdo al confort que experimenta el usuario. Se puede observar en las ciudades, que los espacios exteriores que se encuentran expuestos al sol y que por lo tanto son espacios calurosos, generalmente sin vegetación, son espacios abandonados o poco transitados. Esto además de afectar la imagen urbana propicia problemas de inseguridad, de salud, económicos, entre otros.

Se pueden mencionar dos tipos de estrategias a seguir para mitigar el efecto de la isla de calor urbana. Estrategias preventivas y estrategias correctivas. Las estrategias preventivas serian tales como; tener un plan de desarrollo urbano sostenible, en donde se tenga identificadas las áreas de reserva, pero sobre todo se respete el uso de suelo dentro de la ciudad. Ciudades sostenibles e inclusivas, con suficientes áreas verdes y suelos permeables, propicios para el desarrollo y esparcimiento del ser humano.

Las estrategias correctivas se implementan en espacios o zonas que ya presentan el efecto de isla de calor, insertando elementos que ayuden a mitigar dicho efecto. (Tumini, 2010)

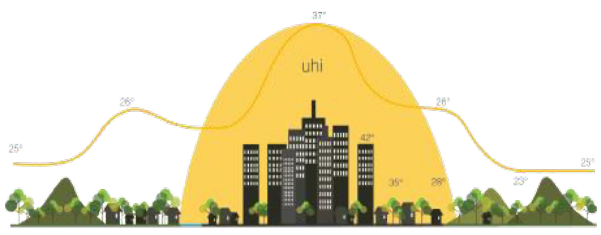

Figura 1: Isla de calor superficial y atmosférica

Las estrategias más utilizadas alrededor del mundo para mitigar la isla de calor urbana superficial, se han propuesto desde enfoques generalmente técnicos en las áreas del urbanismo, la sustentabilidad, la biología y las ciencias ambientales, en donde poco se ha explorado desde el campo creativo del diseño. En este aspecto se considera importante que los procesos de soluciones a problemas complejos se hagan de manera transversal, involucrando a varias disciplinas para poder generar una propuesta de solución integral.

El presente proyecto pretende, incidir en la disminución del efecto de isla de calor urbana superficial mediante el diseño de superficies sensibles que respondan a cambios de temperatura.

Para lo anteriormente expuesto se plantea un escenario con una visión de ciudades con diseños adaptativos que se generan a partir de la inserción de elementos sensibles al entorno, (elementos que respondan a estímulos ambientales) los cuales, convirtiéndose en factores constantes en el diseño a pequeña o gran escala, podrían transformar la manera en la que se modifica el ambiente desde el diseño, hacia un entorno urbano sensible y resiliente ante posibles condiciones ambientales adversas.

\section{METODOLOGÍA}

Para la propuesta y desarrollo del diseño de superficies sensibles se lleva a cabo un proceso iterativo tomando algunos aspectos de Design Thinking como método para la innovación en la solución de problemas (Liu et al., 2011), aplicado a un proceso de Diseño Experimental a partir de la identificación de variables que inciden en la modificación o determinación de un resultado.

En el proceso de diseño se identifican los siguientes aspectos realizados en base al proceso metodológico de design thinking, en este caso no enfocado al usuario si no aplicado mediante la exploración del entorno para definir un problema e idear soluciones que nos llevan a materializar un prototipo.

El proceso de diseño comienza con la identificación de soluciones generadas hasta el momento en aspectos técnicos y desde el campo creativo, para posteriormente realizar una exploración morfológica y material con el fin de generar un prototipo de superficie.

Se propone el uso de materiales inteligentes, como pigmentos termocrómicos, para lograr la variación del color de una superficie, ante un estímulo por temperatura, con lo cual se puede incidir sobre los cambios en la temperatura superficial. Habiendo identificado los materiales a utilizar se realizan exploraciones para la definición morfológica del prototipo, con lo cual se busca que al igual que la variación cromática incida sobre la temperatura, la definición morfológica por variaciones angulares controle la cantidad de superficie que se expone a la incidencia solar de acuerdo a la orientación y ubicación en donde se aplique.

Los prototipos físicos y digitales, generados a partir de este proceso, son sometidos a pruebas de temperatura superficial para comprobar su capacidad de reacción ante dichos cambios, esto como parte de la etapa de evaluación, para posteriormente redefinir y comenzar otro proceso iterativo a partir de la información obtenida.

\section{EXPERIMENTACIÓN}

Se propone la exploración del concreto ya que es uno de los principales materiales utilizados en las ciudades en la actualidad (Naik, 2008), y el cual cuenta con las posibilidades materiales para generar un cambio en las superficies. A pesar de haberse empleado desde hace varios siglos, en la contemporaneidad continúa siendo un material cuya exploración expone múltiples áreas de oportunidad, donde se generan nuevas posibilidades de diseño.

Se modificarán las condiciones iniciales del material mediante la exploración con pigmentos, pigmentos naturales, pigmentos termocrómicos, así como con fibras naturales para intentar modificar su capacidad térmica y de reflectancia. Se espera en algún punto poder modificar las condiciones de un elemento artificial a uno con posibilidades de cambio y respuesta al entorno.

\section{EXPERIMENTO PILOTO CON COLOR}

Con este experimento se pretendía identificar la incidencia en la modificación de la temperatura superficial de un prototipo mediante la exploración material con concreto, pigmentos de tierras naturales de tres colores diferentes, a 
partir de una forma geométrica básica, con la cual se pueda generar una superficie.

Se fabricaron tres prototipos tres prototipos, el prototipo denominado como a1, con pigmento amarillo, el prototipo a2 con pigmento rojo y el prototipo a3, con pigmento azul. Posteriormente se midieron las condiciones térmicas superficiales iniciales de cada prototipo, así como las condiciones térmicas superficiales de cada prototipo, después de haber sido expuestos a la radiación solar, en condiciones específicas y homogéneas para cada experimento. Con esto se identificaron las variaciones térmicas superficiales de cada prototipo de acuerdo a su variación cromática.

A partir de registros y análisis teóricos realizados, se puede deducir que el color más eficiente para una superficie es un color claro, por lo que se esperaba que el color amarillo resultaría con mejores resultados en la eficiencia térmica superficial. (Santamouris, 2016)

En este primer experimento los resultados que se buscaban estuvieron enfocados a la capacidad térmica de prototipo por sus cualidades específicamente cromáticas, habiendo sido fabricados con cualidades materiales similares, por lo que se define una forma geométrica simple, la cual, en base a resultados se podrá complejizar para futuros experimentos.

Diseño y fabricación de molde de poliestireno para la fabricación de los prototipos de concreto (Figura 2 y 3 ).
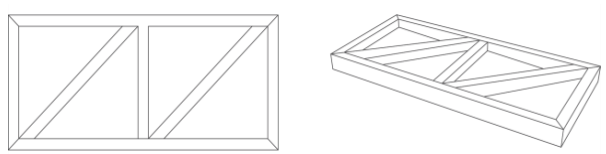

Figura 2: Diagramas de diseño de molde.

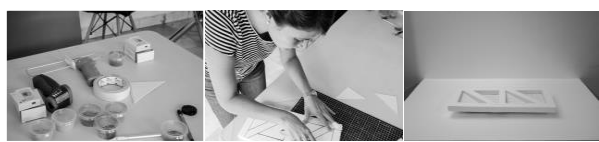

Figura 3: Proceso de fabricación de molde de poliestireno.

Para el experimento piloto se realizó una mezcla homogénea utilizando los agregados convencionales para el concreto, a esta mezcla se le agregó un pigmento de color, los colores que se utilizaron fueron, azul, amarillo y rojo (Figuras 4-8). La mezcla se colocó en un molde con la condición morfológica definida como una geometría básica.

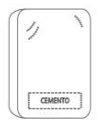

cemento

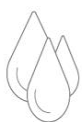

agua

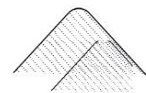

arena

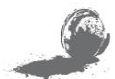

pigmento amarillo
Figura 4: Materiales para la fabricación del prototipo a1

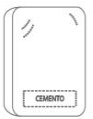

cemento

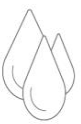

agua arena

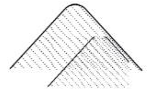

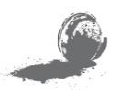

pigmento rojo
Figura 5: Materiales para la fabricación del prototipo a2

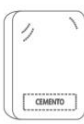

cemento

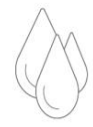

agua

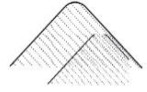

arena

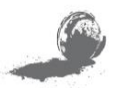

pigmento azul
Figura 6: Materiales para la fabricación del prototipo a3
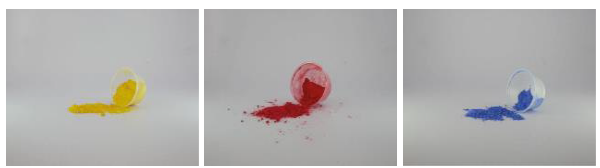

Figura 7: Pigmentos de tierras naturales utilizados para la coloración de los prototipos.
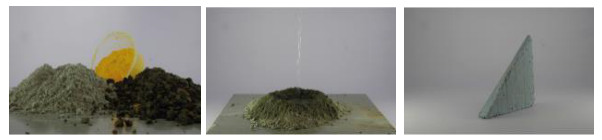

Figura 8: Pigmentos y agregados para la fabricación de los prototipos.

Una vez fabricados los prototipos se procedió a medir la temperatura superficial (Figura 9).

\section{Proceso 1}

- Calibrar termómetro de infrarrojo

- Colocar los tres prototipos fabricados sobre una superficie de poliestireno a la sombra por una hora (mes de mayo)

- Medir temperatura superficial de cada prototipo con termómetro manual de infrarrojo (IRT), colocado apuntando hacia abajo sobre la superficie del prototipo a $10 \mathrm{~cm}$.

\section{Proceso 2}

\section{- Calibrar termómetro de infrarrojo}

- Colocar los tres prototipos fabricados sobre una superficie de poliestireno expuestos a la radiación solar por una hora. Hora de exposición 15:10 - 14:10 (mes de mayo)

- Medir temperatura superficial de cada prototipo con termómetro manual de infrarrojo (IRT), colocado apuntando hacia abajo sobre la superficie del prototipo a $10 \mathrm{~cm}$ después de haber sido expuestos a la radiación solar.
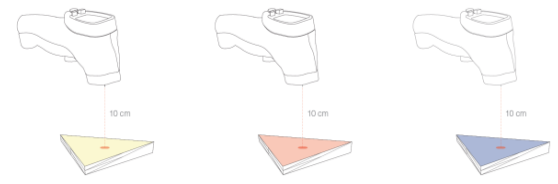

Figura 9: Termómetros infrarrojo (Extech 42510) colocados a $10 \mathrm{~cm}$ de separación de cada prototipo. 


\section{RESULTADOS EXPERIMENTO PILOTO CON COLOR}

Después de haber medido la temperatura superficial de cada prototipo físico fabricado, siendo la única variable de diferenciación, entre cada prototipo, el color, se obtuvieron los siguientes resultados:

Temperatura superficial

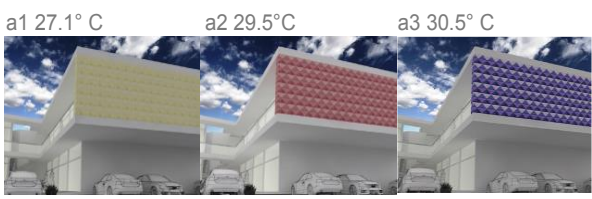

Figura 10: Imágenes representativas de las superficies medidas, correspondiente a cada color utilizado.

La superficie amarilla del prototipo a1 presentó una temperatura de $27.1^{\circ} \mathrm{C}$, siendo la temperatura más baja en relación a la superficie roja del prototipo a2 con una temperatura superficial de $29.5^{\circ} \mathrm{C}$ y la superficie azul del prototipo a3, con una temperatura de $30.5^{\circ} \mathrm{C}$, siendo esta ultima la temperatura superficial más alta registrada en este primer experimento piloto (Figura 10)

De este experimento se concluye que, si el entorno en el que insertamos elementos artificiales estáticos fuera también estático, podríamos elegir alguno de los prototipos explorados de acuerdo a la temperatura que se considere adecuada para un lugar y un tiempo específico con la finalidad de obtener la temperatura superficial deseada. Sin embargo, en un entorno cambiante, por condiciones ambientales, deberíamos tener prototipos también cambiantes y adaptativos o responsivos ante los estímulos externos.

En relación a esto, se busca entonces, explorar el diseño de superficies sensibles a dichos estímulos del entorno, mediante la utilización de materiales inteligentes. Para lo cual, se realizó, posterior a la siguiente exploración con fibras, la experimentación con pigmentos termocrómicos para generar un prototipo que responda a las variaciones ambientales de un entorno e incluso que pueda responder al calor antropogénico.

\section{EXPERIMENTO PILOTO CON FIBRAS}

Con este experimento se pretendía identificar la modificación térmica superficial de un prototipo mediante la exploración material con concreto y fibras naturales de coco, henequén y agave, a partir de una forma geométrica básica, con la cual se pudiera generar una superficie. (Figura 14).

\section{Objetivos particulares}

- Fabricar tres prototipos, con una fibra distinta cada prototipo.

- Medir las condiciones térmicas superficiales iniciales de cada prototipo.

- Medir las condiciones térmicas superficiales de cada prototipo después de haber sido expuesto a la radiación solar.

- Identificar las variaciones térmicas superficiales de cada prototipo de acuerdo a su variación material.

El experimento se llevó a cabo bajo las mismas condiciones morfológicas al experimento piloto con color.
Para la fabricación de los primeros prototipos con fibras, se realizó una mezcla homogénea utilizando los agregados convencionales para el concreto, a esta mezcla se le agregaron fibras naturales de coco, henequén y agave. (Figuras 11-13).

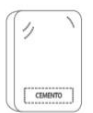

cemento

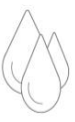

agua

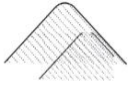

arena

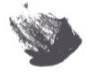

fibra de coco
Figura 11: Materiales para la fabricación del prototipo f1

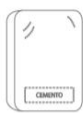

cemento

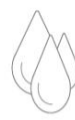

agua

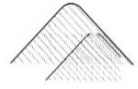

arena

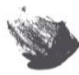

fibra de henequén
Figura 12: Materiales para la fabricación del prototipo f2

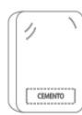

cemento

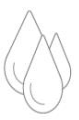

agua

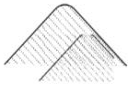

arena

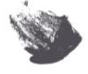

fibra de agave
Figura 13: Materiales para la fabricación del prototipo f3

Las mezclas que se realizaron se vertieron en un molde de poliestireno con las mismas características morfológicas del experimento piloto con color, siendo entonces la única variable de diferenciación ente estos prototipos, el tipo de fibra utilizada entre cada mezcla (Figura 15). Se esperaba identificar una variación térmica superficial entre cada prototipo en relación a cada una de las fibras utilizadas.
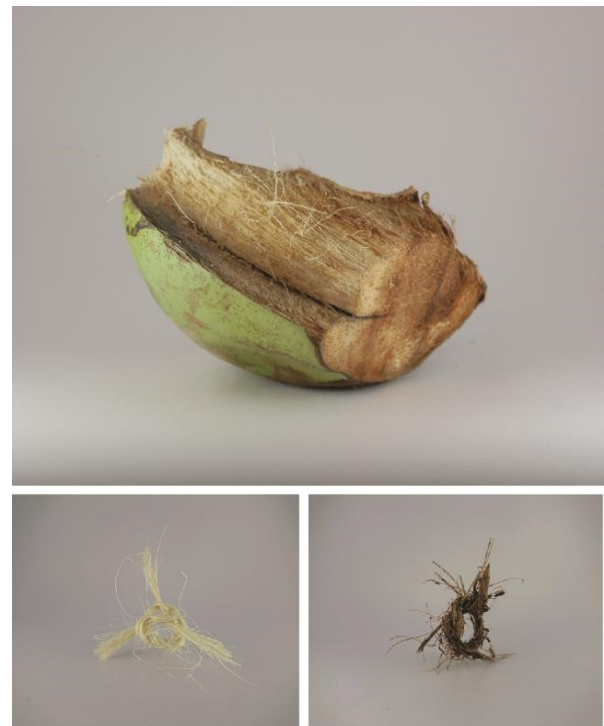

Figura 14: Fibras vegetales de coco, henequén y agave, utilizadas en la fabricación de los prototipos f1, f2 y f3. 
Una vez fabricados los prototipos se procedió a medir la temperatura superficial, siguiendo el mismo protocolo ilustrado en la Figura 9.

\section{Proceso 1}

- Calibrar termómetro de infrarrojo

- Colocar los tres prototipos fabricados sobre una superficie de poliestireno a la sombra durante 1 hora.

- Medir temperatura superficial de cada prototipo utilizando un termómetro manual de infrarrojo (IRT), colocado apuntando hacia abajo a $10 \mathrm{~cm}$ de distancia de la superficie de cada prototipo.

\section{Proceso 2}

- Calibrar termómetro de infrarrojo

- Colocar los tres prototipos fabricados sobre una superficie de poliestireno expuestos a la radiación solar durante 1 hora

- Medir la temperatura superficial de cada prototipo fabricado utilizando un termómetro manual de infrarrojos (IRT) colocado apuntando hacia abajo a $10 \mathrm{~cm}$ de distancia de la superficie de cada prototipo.

\section{RESULTADOS EXPERIMENTO PILOTO CON FIBRAS}

Después de haber medido la temperatura superficial de cada prototipo físico fabricado, siendo la única variable de diferenciación, entre cada prototipo, el tipo de fibra vegetal entre cada mezcla, se obtuvieron los siguientes resultados en relación a la temperatura superficial.

\footnotetext{
- Temperatura superficial del prototipo f1 $22.1^{\circ} \mathrm{C}$

- Temperatura superficial del prototipo f2 $22.3^{\circ} \mathrm{C}$

- Temperatura superficial del prototipo f3 $22.4^{\circ} \mathrm{C}$
}

En relación a los resultados obtenidos, se observa que la temperatura tiene una variación mínima, por lo que de inicio se descarta la posibilidad de que las fibras puedan modificar, de manera significativa, la temperatura superficial.

Sin embargo, las fibras vegetales pueden incidir sobre la capacidad térmica de un elemento, lo cual puede influir en la modificación de una temperatura interior.

Por lo tanto, se podría entonces utilizar el color como un elemento de modificación de temperatura exterior superficial, por niveles de albedo, lo cual incidiría en la mitigación de isla de calor urbana. Por otro lado, las fibras se pueden utilizar como un elemento que incide en la modificación de temperatura al interior de un espacio, por transferencia de calor, al mejorar la capacidad térmica de un elemento aplicado a una superficie o que actué como superficie.
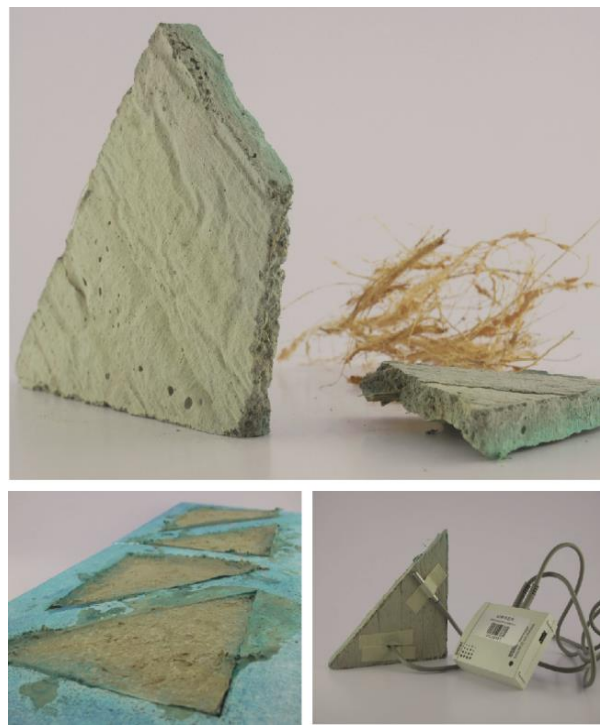

Figura 15: Prototipo f1 de concreto con fibra de coco, molde con prototipos de concreto con fibras vegetales y preparación de prototipo de concreto para medir temperatura con sensor $\mathrm{HOBO}$

\section{EXPERIMENTO PILOTO CON PIGMENTOS TERMOCRÓMICOS}

Con base en los objetivos iniciales del proyecto de investigación, se plantea el escenario en donde el prototipo ideal para una superficie deberá responder a un estímulo del entorno, en este caso, a la variación térmica ambiental.

En el experimento piloto se observa que el color amarillo, del prototipo a1, tiene los resultados más eficientes al marcar una temperatura por debajo de los prototipos a2 y a3, habiendo sido expuesto a la misma cantidad de incidencia solar y estando bajo condiciones similares a las del resto de los prototipos. Por lo que un prototipo más eficiente seria aquel que durante las horas de mayor incidencia solar mantuviera un color claro, y este fuera adquiriendo un color oscuro en las horas de menor incidencia solar. Para lograr estos cambios en respuesta al entorno se propuso la utilización de pigmentos termocrómicos, con los cuales se esperaba poder controlar las variaciones cromáticas de acuerdo a una temperatura determinada (Christie Robert, Robertson Sara, 2007)

Para la realización de este experimento de diseño, entonces, se utilizaron pigmentos termocrómicos con la finalidad de controlar la variación del color, y por lo tanto de la temperatura generada en una superficie.

Además de la utilización de los pigmentos termocrómicos, se fabricaron prototipos con variaciones angulares superficiales, lo cual incidiría al mismo tiempo que la variación cromática, en la cantidad de absorción de energía radiante en relación a la cantidad de superficie expuesta. (Figuras $16-19$ ). 


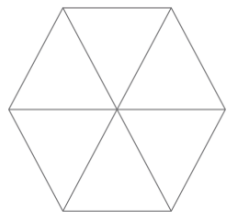

vista superior

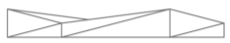

vista latera

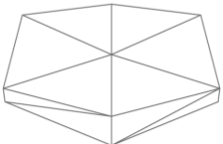

perspectiva

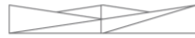

vista frontal
Figura 16: Prototipo af1 con elevación angular de $8^{\circ}$ para obtener mayor superficie de absorción de energía radiante.

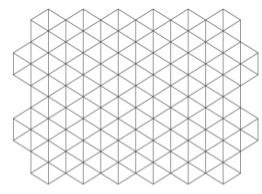

vista superior

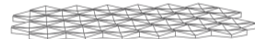

perspectiva

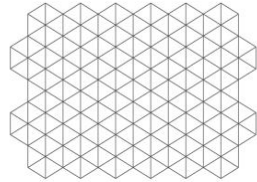

vista superior

\section{Dorstorstors}

vista lateral

vista frontal

Figura 19: Superficie configurada con la aplicación del prototipo af2.
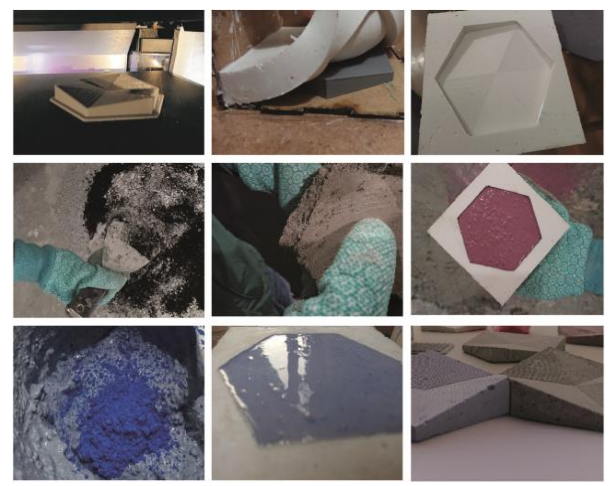

Figura 17: Superficie configurada con la aplicación del prototipo af1.

Los prototipos se diseñaron utilizando herramientas digitales, posteriormente se llevó a cabo un proceso de fabricación digital por adición (impresión 3D con PLA). Se utilizaron las impresiones 3D generadas la fabricación de manera análoga un molde de silicón, el cual fue utilizado para el colado de los prototipos físicos de concreto af1 y af2 (Figura 20).

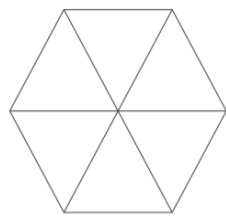

vista superior

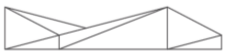

vista lateral

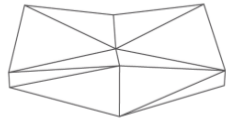

perspectiva

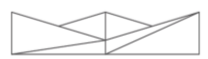

vista frontal

Figura 20: Fabricación de prototipos termocrómicos. Proceso de fabricación digital de prototipo de PLA y fabricación análoga de molde.

Una vez fabricado el molde se procedió con la fabricación de los prototipos a base de una mezcla homogénea de cemento blanco, agua, arena y pigmento termocrómico azul reversible a $31^{\circ}$. En este experimento la variable de diferenciación fue la modificación angular de la superficie (Figura 20).

Habiendo fabricado los prototipos se procedió con el protocolo para medir la temperatura superficial del prototipo af2 siguiendo el mismo protocolo ilustrado en la Figura 9.

Proceso 1

- Calibrar termómetro de infrarrojo

- Colocar el prototipo fabricado af2 sobre una superficie de polisetireno a la sombra.

- Medir temperatura superficial del prototipo utilizando un termómetro manual de infrarrojo (IRT), colocado apuntando hacia abajo a $10 \mathrm{~cm}$ de distancia del prototipo para conocer la temperatura inicial.

\section{Proceso 2}

- Calibrar termómetro de infrarrojo

- Colocar prototipo af2 fabricado sobre una superficie de poliestireno expuestos a la radiación solar por siete horas. Hora de exposición 12:00- 19:00

- Medir la temperatura superficial del prototipo, con un termómetro manual de infrarrojos (IRT) cada hora, colocado apuntando hacía el piso a $10 \mathrm{~cm}$ de distancia de cada prototipo. 


\section{RESULTADOS EXPERIMENTO CON PIGMENTOS TERMOCRÓMICOS}

Después de haber medido la temperatura superficial del prototipo físico fabricado af2, se obtuvieron las siguientes variaciones en la temperatura superficial en relación al cambio cromático que se generó sobre la superficie. (Figuras 21 y 22) $25^{\circ} \mathrm{C}$

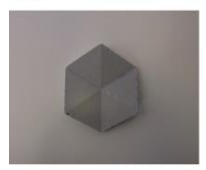

$28^{\circ} \mathrm{C}$

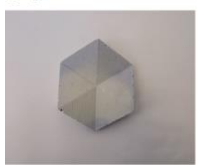

$26^{\circ} \mathrm{C}$

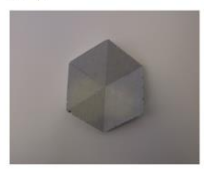

$29^{\circ} \mathrm{C}$

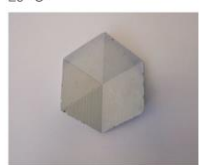

$27^{\circ} \mathrm{C}$

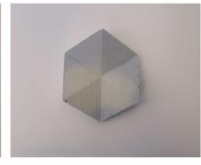

$31^{\circ} \mathrm{C}$

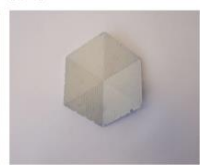

Figure 21: Imágenes del cambio cromático por elevación de temperatura superficial.

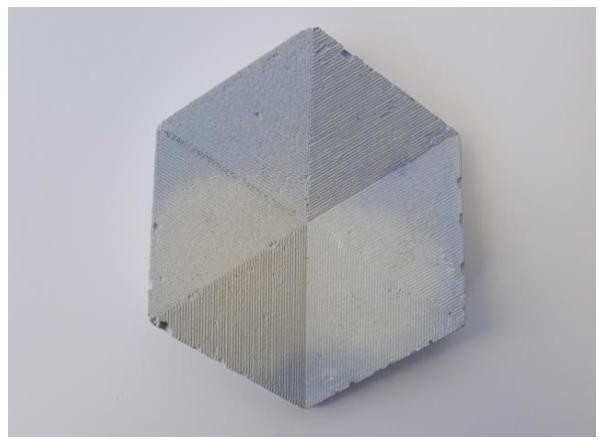

Figura 22: Prototipo fabricado con pigmento termocrómico reversible azul. Temperatura de cambio $31^{\circ} \mathrm{C}$

\section{EXPLORACIONES MORFOLÓGICAS}

Después de haber controlado el color de la superficie con pigmentos termocrómicos se continuó explorando aspectos morfológicos. Se pretende que al igual que el color, la forma de la superficie pueda controlarse para la fabricación de prototipos aplicables a diferentes entornos. (Figura 23)

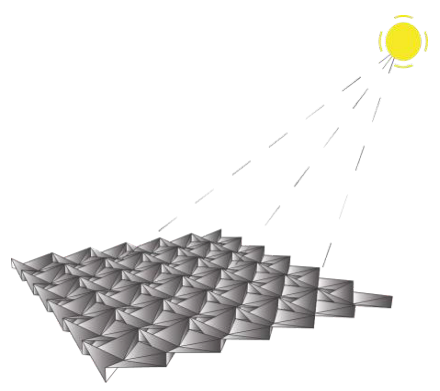

Figura 23: Superficie con variación angular.
En el proceso de la definición de la forma se han logrado identificar algunos aspectos morfológicos importantes para la fabricación de las piezas con eficiencia térmica superficial. En este caso de acuerdo a valores angulares se propone un prototipo que pueda modificarse en dichos valores de manera sencilla y rápida, lo cual nos permitirá modificar el prototipo, de manera digital, de acuerdo a la incidencia solar a la que vaya a ser expuesta la superficie para posteriormente fabricar el prototipo.

En la Figura 24 se observa la definición para generar una superficie con la posibilidad de modificar los parámetros de su elevación angular mediante la variación en los valores de los factores de movimiento, tanto en los ejes horizontales como verticales. Al modificar estos valores se puede controlar la cantidad de calor que puede absorber o reflejar cada superficie, la cual puede ser diferente entre cada superficie.

Se encuentra entonces que el diseño morfológico con posibilidad variable de una superficie, puede aprovecharse para controlar la capacidad de absorción o reflectante de la energía radiante, lo cual podría incidir al mismo tiempo que la variación cromática en la disminución del efecto de isla de calor urbana.

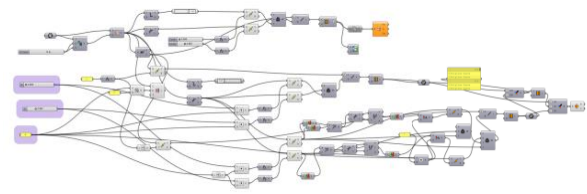

Figura 24: Definición realizada en Grasshopper 3D para Rhinoceros $3 \mathrm{D}$, con la cual se controlan los parámetros angulares y espesores del prototipo con el que se configura la superficie.

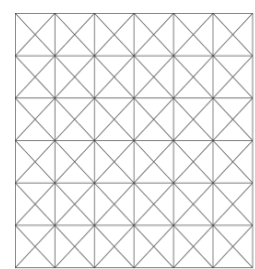

vista superior

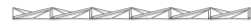

vista lateral

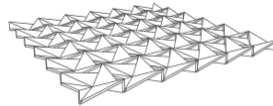

perspectiva

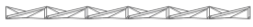

vista frontal
Figura 225: Exploración de los parámetros angulares y espesores de superficies, generada en Rhinoceros a partir de la definición realizada con Grasshopper 3D.

\section{CONCLUSIONES}

En el problema de diseño inicialmente planteado se visualizan ciudades como generalmente las pensamos de manera tradicional, en donde existe una planeación y un diseño, que se anticipa a las necesidades sociales, la realidad es que vivimos en entornos que se encuentran en 
constante cambio, por lo que resulta difícil poder solucionar problemas actuales y futuros con elementos artificiales estáticos insertados en el medio natural. En la actualidad, además de los aspectos estéticos y funcionales, es importante considerar características de resiliencia social, cultural y ambiental. La presente investigación de manera particular se ha centrado en explorar e identificar variables que inciden en la generación de ambientes responsivos.

Particularmente los resultados de exploraciones con fibras demostraron que estas no modifican de manera significativa la temperatura superficial. En contraste, se logró identificar que el pigmento termocrómico reversible actúa de manera significativa, demostrando la capacidad de adaptabilidad modificando la intensidad del color al exponerse de manera natural a la incidencia de la radiación solar. Un prototipo aplicado con un color azul para condiciones en donde la superficie no este expuesta al sol, se torne blanca al comenzar a calentarse, ya que como se ha estudiado, los colores claros tienen un mayor índice de reflectancia y por lo tanto la capacidad de absorción de calor del prototipo disminuye al modificarse, de un color oscuro a uno claro.

Los resultados obtenidos con la realización de este proyecto muestran que las superficies pueden ser manipuladas para convertirlas en elementos que respondan al entorno, en este caso con la modificación material, cromática y morfológica, lo cual puede incidir en la mitigación de la elevación de la temperatura superficial que genera el efecto de isla de calor urbana. Estos resultados abren un campo de oportunidad para seguir explorando en este sentido, haciendo posible la aplicación de esta investigación a futuros proyectos académicos y actuales prácticas profesionales.

\section{FUTURA INVESTIGACIÓN}

Las experimentaciones expuestas en este artículo permitieron definir las líneas para futuras exploraciones, las cuales deberán de dirigirse para establecer numéricamente la respuesta del material termocrómico (porcentaje de reflectividad, coeficiente albedo, emisividad) y establecer el impacto del mismo a través de aplicaciones en fachadas, pavimentos y/o cubiertas.

\section{AGRADECIMIENTOS}

Los autores agradecen al Consejo de Ciencia y Tecnología (CONACYT) por su apoyo y patrocinio para la realización de éste proyecto, así como a la Maestría en Diseño Avanzado de la Universidades Michoacana de San Nicolás de Hidalgo. Agradecen también la oportunidad de participar con el presente proyecto de investigación en el Congreso Internacional de la Sociedad Iberoamericana de Grafica Digital a través de la Universidad Pontificia Bolivariana.

\section{REFERENCIAS}

Christie Robert, Robertson Sara, T. S. (2007). Colour: Design \& Creativity. Colour: Design \& Creativity, 1(2), 1-9.

Liu, J., Zhang, M., \& Hu, X. (2011). Understanding Design Thinking: a Process. September, 44-48. https://doi.org/10.1109/CAD/Graphics.2011.56

Naik, T. R. (2008). Sustainability of concrete construction. Practice Periodical on Structural Design and Construction, 13(2), 98-103. https://doi.org/10.1061/(ASCE)10840680(2008)13:2(98)

Rizwan, A. M., Dennis, L. Y. C., \& LIU, C. (2008). A review on the generation, determination and mitigation of Urban Heat Island. Journal of Environmental Sciences, 20(1), 120-128. https://doi.org/10.1016/S1001-0742(08)60019-4

Santamouris, M. (2016). Urban Climate Mitigation Strategies (U. of Athens (ed.)).

Tumini, I. (2010). Estrategias para reducción del efecto isla de calor en los espacios urbanos. Estudio aplicado al caso de Madrid. Congreso Internacional Sobre Edificación Sostenible SB10mad, 1-15. www.sb10mad.com/ponencias/archivos/a/A033.pdf

United Nations. (2018). Las ciudades seguirán creciendo, sobre todo en los países en desarrollo | ONU DAES | Naciones Unidas Departamento de Asuntos Económicos y Sociales. https://www.un.org/development/desa/es/news/population/ 2018-world-urbanization-prospects.html 